\title{
Integrative Bioinformatics Analysis Reveals Potential Target Genes and TNF $\alpha$ Signaling Inhibition by Brazilin in Metastatic Breast Cancer Cells
}

\author{
Adam Hermawan ${ }^{1 *}$, Herwandhani Putri ${ }^{2}$
}

\begin{abstract}
Objective: Metastasis is the most significant cause of morbidity and mortality in breast cancer patients. Previously, a combination of brazilin and doxorubicin has been shown to inhibit metastasis in HER2-positive breast cancer cells. This present study used an integrative bioinformatics approach to identify new targets and the molecular mechanism of brazilin in inhibiting metastasis in breast cancer. Methods: Cytotoxicity and mRNA arrays data were retreived from the DTP website, whereas genes that regulate metastatic breast cancer cells were retreived from PubMed with keywords "breast cancer metastasis". Gene ontology (GO), Kyoto Encyclopedia of Genes and Genomes (KEGG) pathway enrichment, and Drug association analysis were carried out by using WEB-based GEne SeT AnaLysis Toolkit (WebGestalt). Construction of protein-protein interaction (PPI) network analysis was performed by STRING-DB v11.0 and Cytoscape, respectively. The genetic alterations of the potential therapeutic target genes of brazilin (PB) were analyzed using cBioPortal. Results: Analysis of cytotoxicity with the public database of COMPARE showed that brazilin exerts almost the same cytotoxicity in the NCI-60 cells panel showing by similar GI50 value, in which the lowest GI50 value was observed in MDA-MB 231, a metastatic breast cancer cells. KEGG enrichment indicated several pathways regulated by brazilin such as TNF signaling pathway, cellular senescence, and pathways in cancer. We found ten drugs that are associated with PB, including protein kinase inhibitors, TNF $\alpha$ inhibitors, enzyme inhibitors, and anti-inflammatory agents. Conclusion: In conclusion, this study identified eight PB, including MMP14, PTGS2, $A D A M 17, P T E N, C C L 2, P I K 3 C B, M A P 3 K 8$, and $C X C L 3$. In addition, brazilin possibly inhibits metastatic breast cancer through inhibition of TNF $\alpha$ signaling. The study results study need to be validated with in vitro and in vivo studies to strengthen scientific evidence of the use of brazilin in breast cancer metastasis inhibition.
\end{abstract}

Keywords: Metastasis- breast cancer- bioinformatics- TNF $\alpha$ signaling- Brazilin

Asian Pac J Cancer Prev, 21 (9), 2751-2762

\section{Introduction}

Metastasis is the most significant cause of morbidity and mortality in breast cancer patients (Yousefi et al., 2018). The first-line treatment for metastases in breast cancer is chemotherapy (Telli and Carlson, 2009); however, its prolonged use induces side effects, such as cardiotoxicity (Shafei et al., 2017) and suppression of immune system (Larsson et al., 2019). Moreover, the effectivity of chemotherapy in breast cancer treatment decrease due to intrinsic and acquired chemoresistance, which leads to relapse and metastasis (Rivera and Gomez, 2010). Developing a strategy that can overcome the progression and metastasis of breast cancer cells is necessary, including the development of new drugs from natural products.

One compound that has the potential to inhibit metastasis is brazilin, a compound isolated from sappan wood (Caesalpinia sappan L.) (Nirmal et al., 2015). Brazilin was shown to induce apoptosis and inhibit cell proliferation in several cancer cells, including MCF-7 breast cancer cells (Naik Bukke et al., 2018), T24 bladder cancer urinary cells (Zhang et al., 2015), U266 multiple myeloma cells (Kim et al., 2012), U87 glioma cells (Lee et al., 2013), Cal27 head squamous cell carcinoma cells and neck cancer cells (Lee et al., 2013), MG-63 osteosarcoma cells (Lee et al., 2018), and Tca8113 tongue cancer cells (Jia et al., 2019). The combination of brazilin and doxorubicin has also been shown to inhibit metastasis in HER2-positive breast cancer through downregulation of MMP9, MMP2, and Rac1 (Jenie et al., 2018). Breast cancer is a complex disease (Fragomeni et al., 2018) and the mechanisms of metastasis involve many key proteins (Welch and Hurst, 2019), and therefore discover new

${ }^{1}$ Laboratory of Macromolecular Engineering, Department of Pharmaceutical Chemistry, Faculty of Pharmacy, Universitas Gadjah Mada Sekip Utara II, 55281 Yogyakarta, Indonesia. ${ }^{2}$ Cancer Chemoprevention Research Center, Faculty of Pharmacy, Universitas Gadjah Mada Sekip Utara II, 55281 Yogyakarta, Indonesia.*For Correspondence: adam_apt@ugm.ac.id 
molecular targets and their molecular changes of brazilin in metastatic breast cancer cells needs to be done.

This present study used an integrative bioinformatics approach to identify new targets and molecular mechanisms of brazilin in inhibiting metastases in breast cancer. The brazilin target was retrieved from the NCI COMPARE, while the metastatic breast cancer regulatory gene was downloaded from PubMed, which from both we made a Venn diagram consisting of the potential therapeutic target of brazilin against metastatic breast cancer cells (Figure 1). Analysis of protein-protein interaction network, gene ontology, enrichment of the KEGG pathway, and genetic alterations, reveal the targets and molecular mechanisms of brazilin in inhibiting metastatic breast cancer. This present study could be the basis for the development of brazilin as an antimetastatic agent in breast cancer.

\section{Materials and Methods}

\section{Data mining and processing}

Cytotoxic effect and mRNA expression data were collected from the NCI 60 DTP website (http.dtp.nci.nih. gov) (Monks et al., 1997). COMPARE analysis with the public library generates a list of high similarity drugs with brazilin, and a list of mRNA expressions upon brazilin treatment in the NCI 60 cells collection (Mahmoud et al., 2018). The similarity pattern is presented as the Pearson correlation coefficient, with the cutoff value of $<-0.3$ and $>$ 0.3 . Regulatory genes of metastatic breast cancer were retreived from PubMed database with keywords "breast cancer metastasis“.

Gene ontology and KEGG pathway enrichment analysis Gene ontology (GO) and Kyoto Encyclopedia of Genes and Genomes (KEGG) pathway enrichment analysis were carried out by using Overrepresentation Enrichment Analysis (ORA) from WEB-based GEne SeT AnaLysis Toolkit (WebGestalt), (Wang et al., 2017), with $\mathrm{p}<0.05$ as the cutoff value.

\section{Drug association analysis}

Drug association analysis was performed using Overrepresentation Enrichment Analysis (ORA) from WEB-based GEne SeT AnaLysis Toolkit (WebGestalt) with $\mathrm{p}<0.05$ and FDR $<0.05$ was selected as the cutoff value (Wang et al., 2017).

\section{PPI network and hub genes analysis}

PPI network was analyzed with STRING-DB v11.0 (Szklarczyk et al., 2015), with confidence scores $>0.7$ were considered significant. PPI network further was visualized by Cytoscape. Selection of hub genes based on the highest degree score was conducted by CytoHubba plugin (Chin et al., 2014).

\section{Analysis of genetic alterations of the $P B$}

The genetic alterations of the potential therapeutic target genes of brazilin (PB) were analyzed using cBioPortal (Cerami et al., 2012; Gao et al., 2013). The breast cancer study with the highest genetic alterations was chosen for further connectivity analysis with cutoff value of $\mathrm{p}<0.05$.

\section{Results}

\section{Data mining and analysis}

This present study aimed to identify the new molecular targets and mechanism of brazilin in inhibition of metastastic breast cancer cells. Analysis of cytotoxicity with the public database of COMPARE showed that brazilin exerts almost the same cytotoxicity in the NCI-60 cells panel showing a similar GI50 value (Figure 2A). The lowest GI50 value was observed in prostate and breast cancer. Moreover, the GI50 value of brazilin in MDA-MB 231 , metastatic breast cancer cells, was one of the lowest amongst other cell lines (Supplementary Table 1).

Analysis with COMPARE identified 13 standard agents with a correlation with brazilin, either direct or inverse (Supplementary Table 2). Pancratiastatin and S-trityl-Lcysteine are standard drugs with the highest score of the Pearson correlation coefficient, whereas AT-125 (acivicin) and mitindomide are standard drugs with the lowest score of Pearson correlation coefficient (Supplementary Table 2). COMPARE analysis revealed 1249 genes regulated by brazilin (Supplementary Table 3), including 587 and 662 genes with the positive and negative Pearson correlation coefficient, respectively. GLRX3, SMG5, SLC6A4 (with Pearson correlation coefficient of $0.476,0.459,0.455$, respectively) are genes with direct correlation. SLC7A11, IL37, and $P H K B$, with a Pearson correlation coefficient of $-0.592,-0.551$, and -0.547 , respectively, are genes with inverse correlation. Direct correlation shows that the higher mRNA expression, the higher the chemoresistance, while inverse correlation shows that the higher expression of mRNA, the higher the chemosensitivity of the drugs.

Since brazilin exerted high cytotoxicity towards metastatic breast cancer cells, we retrieved 2,263 regulatory genes of metastasis in breast cancer (Figure 2B) Moreover, a Venn diagram between Brazilin target from COMPARE and regulatory genes of metastatic breast cancer, revealed 102 genes, which were then considered as potential targets of brazilin in metastatic breast cancer (PB).

GO, KEGG pathway, and drug association analysis of potential targets of brazilin in metastatic breast cancer

Gene ontology analysis was categorized into biological process, cellular component, and molecular function (Figure 2C). We found that PB was mostly involved in response to stimulus, metabolic process, and cell communication. In addition, the PB was located in the membrane, nucleus, and cytosol, and play a role in the molecular function in protein, ion and nucleic acid binding, as well as enzyme regulator activity. KEGG enrichment indicated several pathways regulated by brazilin (Table 2) such as the TNF signaling pathway, cellular senescence, and pathways in cancer. Several $\mathrm{PB}$ was involved in TNF $\alpha$ signaling pathway, including CASP3, CCL2, CXCL3, MAP3K8, MMP14, PIK3CB, $P T G S 2$, and TNFRSF1A (Supplementary Table 5, Figure 3 ). From drug association analysis, we found ten drugs 
Table 1. KEGG Pathway Enrichment Analysis of the DEGs

\begin{tabular}{|c|c|c|c|}
\hline Gene Set & Description & P Value & FDR \\
\hline hsa04668 & TNF signaling pathway & $1.02 \mathrm{E}-07$ & 0.000033174 \\
\hline hsa04218 & Cellular senescence & 0.000025109 & 0.0025615 \\
\hline hsa04060 & Cytokine-cytokine receptor interaction & 0.000026574 & 0.0025615 \\
\hline hsa04657 & IL-17 signaling pathway & 0.000033405 & 0.0025615 \\
\hline hsa05200 & Pathways in cancer & 0.000039287 & 0.0025615 \\
\hline hsa04933 & AGE-RAGE signaling pathway in diabetic complications & 0.000050078 & 0.0027209 \\
\hline hsa05418 & Fluid shear stress and atherosclerosis & 0.000059126 & 0.0027536 \\
\hline hsa05220 & Chronic myeloid leukemia & 0.000096584 & 0.003861 \\
\hline hsa05206 & MicroRNAs in cancer & 0.00010659 & 0.003861 \\
\hline hsa05163 & Human cytomegalovirus infection & 0.00034585 & 0.011275 \\
\hline hsa05142 & Chagas disease (American trypanosomiasis) & 0.00048594 & 0.014402 \\
\hline hsa04659 & Th17 cell differentiation & 0.00062734 & 0.014837 \\
\hline hsa04931 & Insulin resistance & 0.00062734 & 0.014837 \\
\hline hsa04932 & Non-alcoholic fatty liver disease (NAFLD) & 0.00063716 & 0.014837 \\
\hline hsa04115 & p53 signaling pathway & 0.00069172 & 0.015033 \\
\hline hsa04390 & Hippo signaling pathway & 0.00077556 & 0.015802 \\
\hline hsa04217 & Necroptosis & 0.0010456 & 0.018937 \\
\hline hsa04630 & JAK-STAT signaling pathway & 0.0010456 & 0.018937 \\
\hline hsa04380 & Osteoclast differentiation & 0.0015995 & 0.027443 \\
\hline hsa04068 & FoxO signaling pathway & 0.0018718 & 0.030511 \\
\hline hsa05222 & Small cell lung cancer & 0.0020866 & 0.032393 \\
\hline hsa04010 & MAPK signaling pathway & 0.0023689 & 0.035103 \\
\hline hsa05215 & Prostate cancer & 0.0026322 & 0.037308 \\
\hline hsa05161 & Hepatitis B & 0.002903 & 0.039432 \\
\hline hsa04625 & C-type lectin receptor signaling pathway & 0.0035609 & 0.046434 \\
\hline hsa05230 & Central carbon metabolism in cancer & 0.0037962 & 0.047599 \\
\hline
\end{tabular}

that are associated with brazilin, including protein kinase inhibitors, TNF $\alpha$ inhibitors, enzyme inhibitors, and antiinflammatory agents (Table 2).

\section{PPI network and hub genes analysis}

A PPI network were constructed from 102 proteins (confidence level of 0.4) consists of 99 nodes, 233 edges, PPI enrichment value of $<1.10 \mathrm{e}-16$, and average local clustering coefficient of 0.396 ) (Figure 4A). The top 20 of highest degree score genes were revealed, including, PTEN, CASP3, PTGS2, and ADAM17 (Figure 4B, Table 3).

\section{Analysis of genetic alterations of the $P B$}

Eight potential target genes of brazilin (PB) were analyzed using cBioportal to explore their genomic alterations across breast cancer studies. CCL2, $M A P 3 K 8$, $M M P 14, P I K 3 C B$, and $C X C L 3$ were selected from KEGG pathway enrichment analysis. PTGS2, MMP14, ADAM17, $P T E N, C C L 2$, and CXCL3 were selected based on the highest degree score using CytoHubba. A study, namely the METABRIC (Lefebvre et al., 2016), was selected for further analysis (Figure 5A). Genetic alterations for each target genes were found as MMP14 (1.2\%), ADAM17 (1.2\%), PIK3CB (1.6\%), MAP3K8 (1.6\%), CXCL3 (1.9\%), CCL2 (2\%), PTEN (7\%), and PTGS2
$(22 \%)$ (Figure 5B).

\section{Discussion}

This study was aimed to identify the new targets and molecular mechanisms of brazilin in inhibition of metastases in breast cancer using an integrative bioinformatics approach. Analysis using COMPARE showed that brazilin had the lowest GI50 value amongst other cells in MDA-MB 231 cells, highly metastatic breast cancer cells (Liu et al., 2019). This phenomenon revealed the high potency of brazilin as an anticancer agent against metastatic breast cancer.

Analysis with COMPARE identified 13 standard agents that have a correlation with brazilin, in which pancratiastatin and S-trityl-L-cysteine are standard drugs with the highest score of Pearson correlation coefficient, whereas AT-125 (acivicin) and mitindomide are standard drugs with the lowest score of Pearson correlation coefficient. The high value of the Pearson correlation coefficient means, the higher the cytotoxicity of a standard compound, the higher the cytotoxicity of brazilin. Vice versa, the low value of the Pearson correlation coefficient means the higher the cytotoxicity of a standard compound, the lower the cytotoxicity of brazilin. A previous study showed that pancratistatin, 
Table 2. Top 10 Drug Association Analysis

\begin{tabular}{llcc}
\hline Gene Set & Description & P value & FDR \\
\hline PA164712838 & Interleukin inhibitors & $6.63 \mathrm{E}-11$ & $1.21 \mathrm{E}-07$ \\
PA164713204 & Protein kinase inhibitors & $3.57 \mathrm{E}-09$ & 0.000003264 \\
PA164713366 & Tumor necrosis factor alpha (TNF-alpha) inhibitors & $7.16 \mathrm{E}-08$ & 0.000037631 \\
PA166049190 & flufenamic acid & $8.24 \mathrm{E}-08$ & 0.000037631 \\
PA164712732 & Enzyme inhibitors & $1.08 \mathrm{E}-07$ & 0.000039341 \\
PA164712734 & Enzymes & $1.71 \mathrm{E}-07$ & 0.000051958 \\
PA164712839 & Interleukins & $1.76 \mathrm{E}-06$ & 0.00045974 \\
PA164774763 & latanoprost & 0.000016719 & 0.0038181 \\
PA164712458 & Antiinflammatory Agents & 0.000021643 & 0.0043934 \\
PA450744 & Oxygen & 0.000027381 & 0.0044111 \\
\hline
\end{tabular}

alkaloid compound isolated from Amaryllidaceae plant, promotes apoptosis and autophagy in DU145 and $\mathrm{LNCaP}$ metastatic prostate cancer cells (Griffin et al., 2011). Another study demonstrated that S-trityl-Lcysteine isolated from garlic is a novel $\mathrm{Eg} 5$ inhibitor for chemotherapy against neuroblastoma cells (Wu et al., 2018). Acivicin, an antibiotic, and chemotherapy isolated from Streptomyces sviceus (Poster et al., 1981). A phase II clinical trial of acivicin has been performed against advanced metastatic breast cancer patients (Fleishman et al., 1983). Moreover, a combination of acivicin and cisplatin inhibits metastasis in B16F10 melanoma cells (Roy and Maity, 2007). Another study showed that mitindomide free and liposomal drugs showed cytotoxicity against L1210 leukemic cells (Sampedro et al., 1994). Moreover, mitindomide exerts cytotoxicity through inhibition of topoisomerase II (Hasinoff et al., 1997). Accordingly, brazilin probably acts as pancriastatin in inhibiting metastatic breast cancer cells.

KEGG pathway enrichment analysis revealed that brazilin targets the TNF signaling pathway in inhibition of metastatic breast cancer cells. Tumor necrosis factor-alpha $(\mathrm{TNF} \alpha)$ is a cytokine that regulates various biological processes of cancer, including inflammation (Scheff et al., 2017), cell proliferation and apoptosis (Lyu et al., 2017), progression and metastasis (Ham et al., 2016), and chemoresistance (Zhang et al., 2018). The signaling starts when TNF $\alpha$ binds into its receptor, TNF $\alpha$ RI (a member of TNF $\alpha$ R family together with TNF $\alpha$ RII), and leads to receptor trimerization and the recruitment of adaptor protein and TNF $\alpha \mathrm{R}$ associated factor, followed by activation of IKK and transactivation of NFkB (Wu and Zhou, 2010).

$\mathrm{TNF} \alpha$ signaling pathway regulates metastasis in breast cancer through several mechanisms. Activation of NFkB by TNF $\alpha$ promotes breast cancer by promoting

Table 3. Top 20 Hub Genes Based on Highest Score Degree, Analyzed by CytoHubba

\begin{tabular}{lllc}
\hline Rank & Gene Symbol & Gene Name & Score \\
\hline 1 & PTEN & Phosphatase and tensin homolog & 26 \\
2 & CASP3 & Caspase-3 & 25 \\
3 & PTGS2 & Prostaglandin G/H synthase 2 & 19 \\
3 & CCL2 & C-C motif chemokine 2 & 19 \\
5 & LEP & Leptin & 16 \\
6 & IL17A & Interleukin-17A & 15 \\
7 & SMAD3 & Mothers against decapentaplegic homolog 3 & 14 \\
8 & TNFRSF1A & Tumor necrosis factor receptor superfamily member 1A & 13 \\
8 & SOCS3 & Suppressor of cytokine signaling 3 & 13 \\
10 & FOXP3 & Forkhead box protein P3 & 12 \\
10 & MMP1 & Interstitial collagenase & 12 \\
10 & CDKN2A & Cyclin-dependent kinase inhibitor 2A & 12 \\
13 & CDH5 & Cadherin-5 & 10 \\
13 & SELE & E-selectin & 10 \\
15 & HSP90AB1 & Heat shock protein HSP 90-beta & 9 \\
15 & TGFBR1 & TGF-beta receptor type-1 & 9 \\
15 & MDM2 & E3 ubiquitin-protein ligase Mdm2 & 9 \\
15 & MMP14 & Matrix metalloproteinase-14 & 9 \\
15 & YWHAZ & 14-3-3 protein zeta/delta & 9 \\
\hline
\end{tabular}

2754 Asian Pacific Journal of Cancer Prevention, Vol 21 


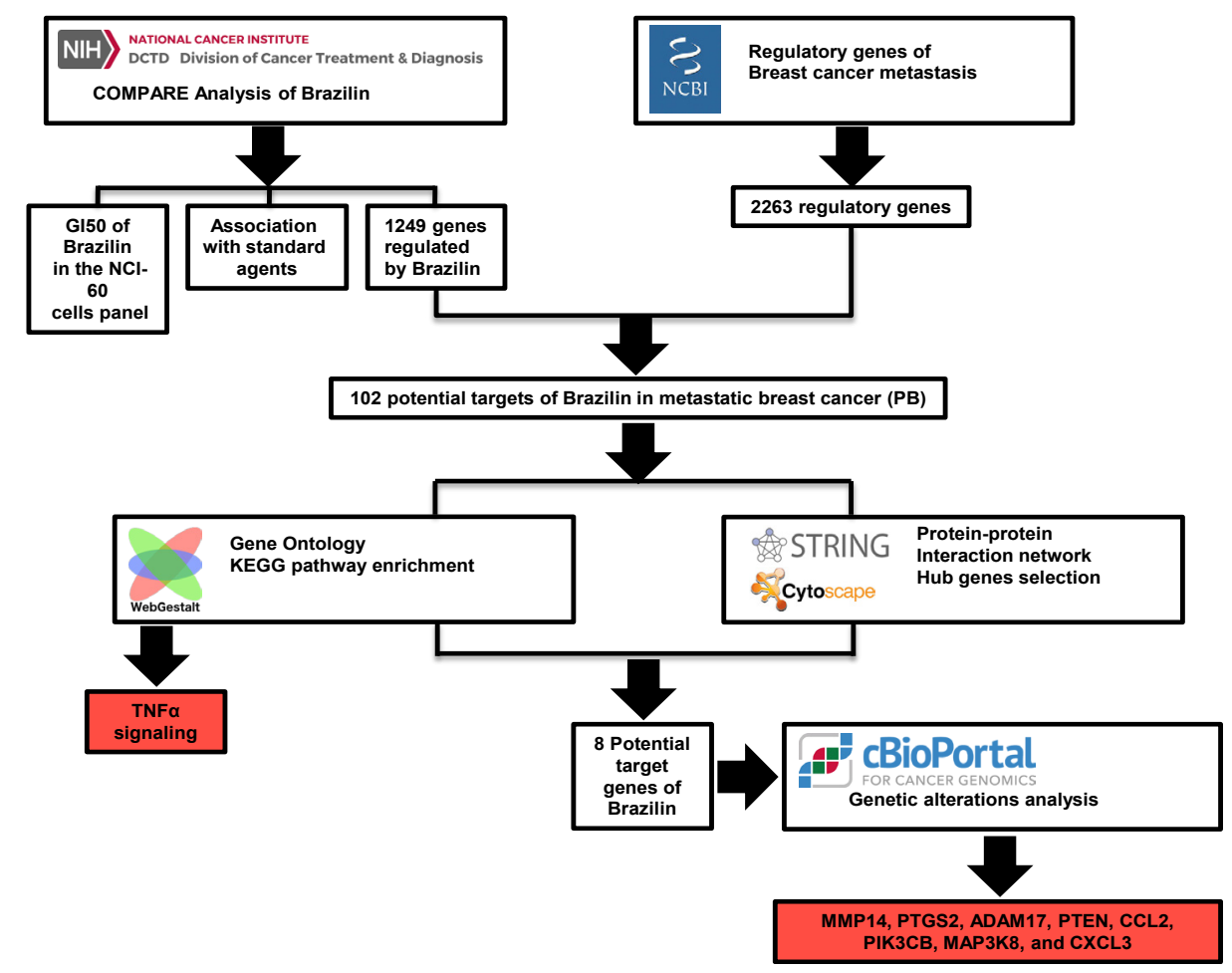

Figure 1. Flowchart of the Study

cell proliferation and migration in breast cancer cells (Kawabata et al., 2017). TNF $\alpha$ signaling also activates the ERK/JNK/p38 pathway leading to migration and invasion in colon cancer cells (Zhao and Zhang, 2018), and breast cancer cells (Qiu et al., 2019). Another study demonstrated that $\mathrm{TNF} \alpha$ activates mesenchymal stromal cells that leads to metastasis in breast cancer through the recruitment of CXCR2+ neutrophils (Yu et al., 2017). In addition, TNF $\alpha$ contributes to the aggressive properties of triple-negative breast cancer cell lines via the upregulation of TNFAIP3(A20) (Lee et al., 2019). Recently, TNF $\alpha$ involves in the invasion and metastasis of breast cancer cells by promoting crosstalk between mitochondria and lysosomal function (Singh et al., 2019). The results of KEGG pathway enrichment analysis is supported by the results of drug association analysis, which showed that potential therapeutic target of brazilin against metastatic breast cancer cells (PB) are associated with tyrosine kinase inhibitors and TNF $\alpha$ inhibitors (Supplementary Table 6). Nevertheless, these findings need to be validated further with in vitro and in vivo studies.

In the following section, we will discuss each gene target, its potential as a target of brazilin, and its relationship to TNF $\alpha$ signaling. MMP14 encodes matrix metalloproteinase 14 (Mmp14) or also known as membrane type-1 matrix metalloproteinase (MT1-MMP), an enzyme that plays a role in the breakdown of extracellular matrix in invasion, metastasis, and angiogenesis (Shuman Moss et al., 2012). Overexpression of MMP14 in serum was found in patients with invasive gastric cancer (Kasurinen et al., 2018). A recent study revealed that MMP14 is a biomarker of poor prognosis in patients with colorectal cancer (Cui et al., 2019). MMP19 plays a role in the TNF $\alpha$ signaling pathway. TNF $\alpha$ increased the expression of MMP14 during fracture healing (Lehmann et al., 2005). Moreover, TNF $\alpha$ activates the MAPK/ERK signaling pathway and
A

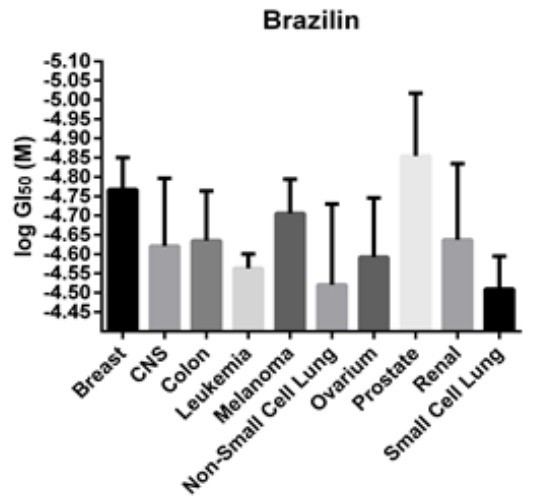

B

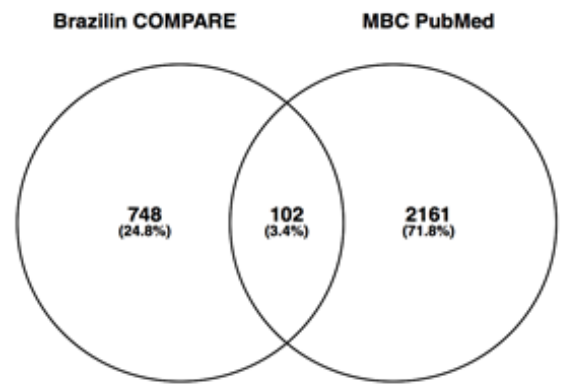

Figure 2. (A). Cytotoxicity (GI50 value) of brazilin in the NCI-60 cells. (B). A Venn diagram between the mRNA array of brazilin (from COMPARE) and genes related to breast cancer metastasis (from PubMed). (C). GO enrichment analysis of Brazilin targets in metastatic breast cancer, analyzed by WebGestalt. 


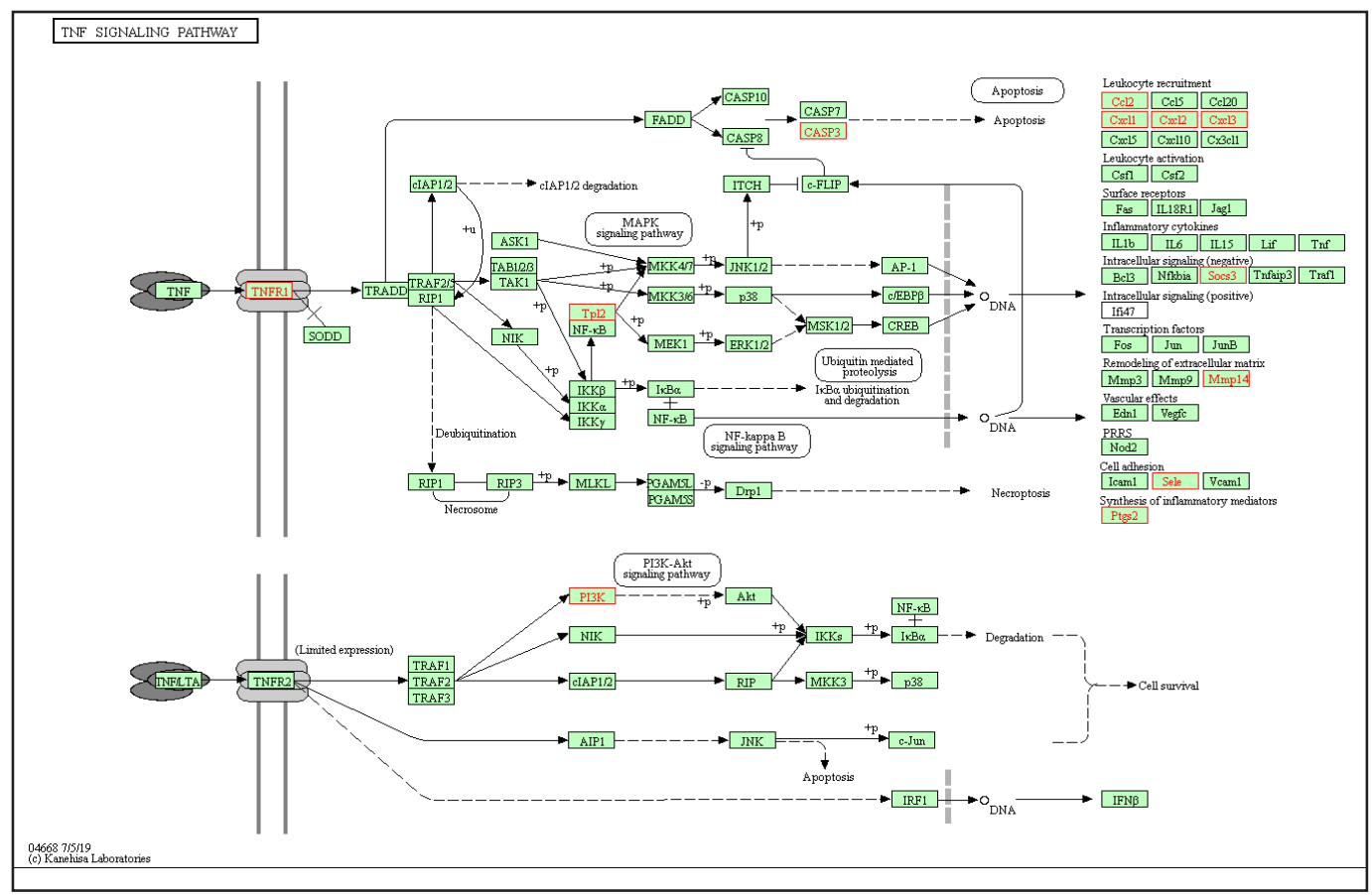

Figure 3. TNF Signaling Pathway (Retrieved from KEGG) as One of the Pathways of Brazilin Targets in Metastatic Breast Cancer

subsequently promotes the migration of breast cancer cells through the upregulation of MMP9, MMP14, and MMP2 in the lipid rafts (Wolczyk et al., 2016). Nevertheless, the role of MMP14 and TNF $\alpha$ signaling upon brazilin treatment needs to be explored further.

PTGS2 encodes prostaglandin-endoperoxide

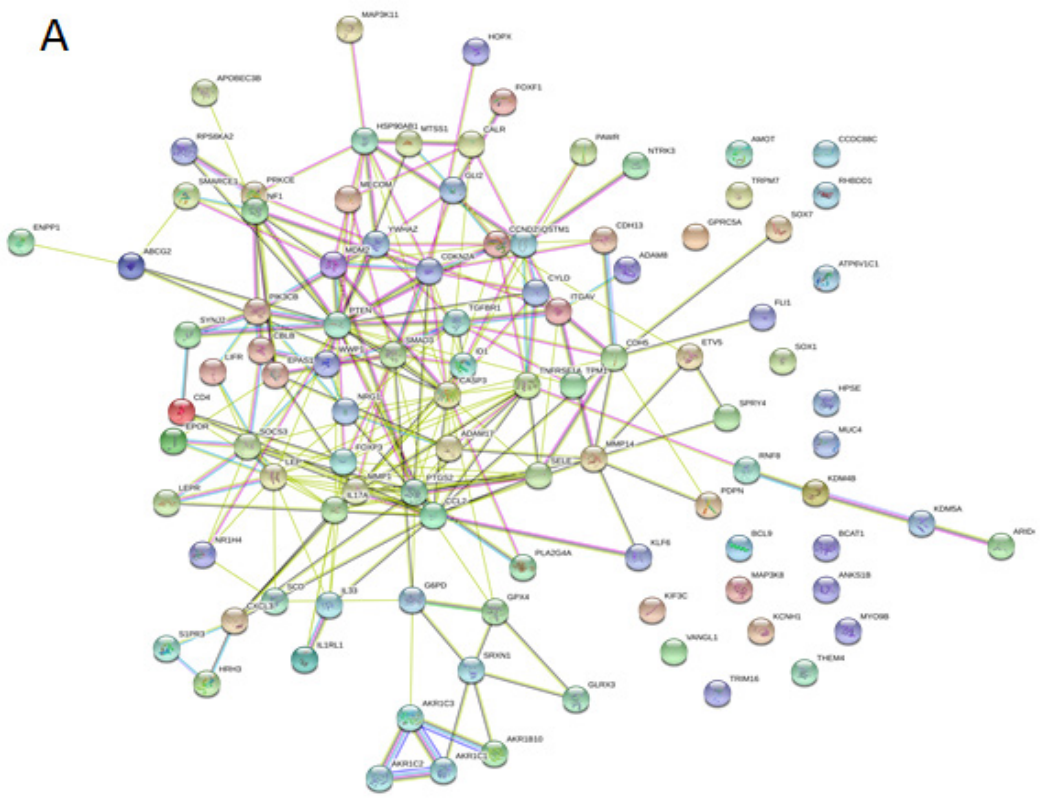

B

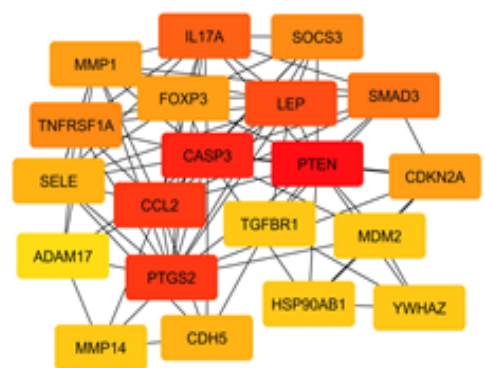

Figure 4. (A). Protein-protein interaction network of Brazilin targets in metastatic breast cancer, analyzed by STRING. (B). Top 20 hub genes based on highest degree score, analyzed by Cytohubba. 
A
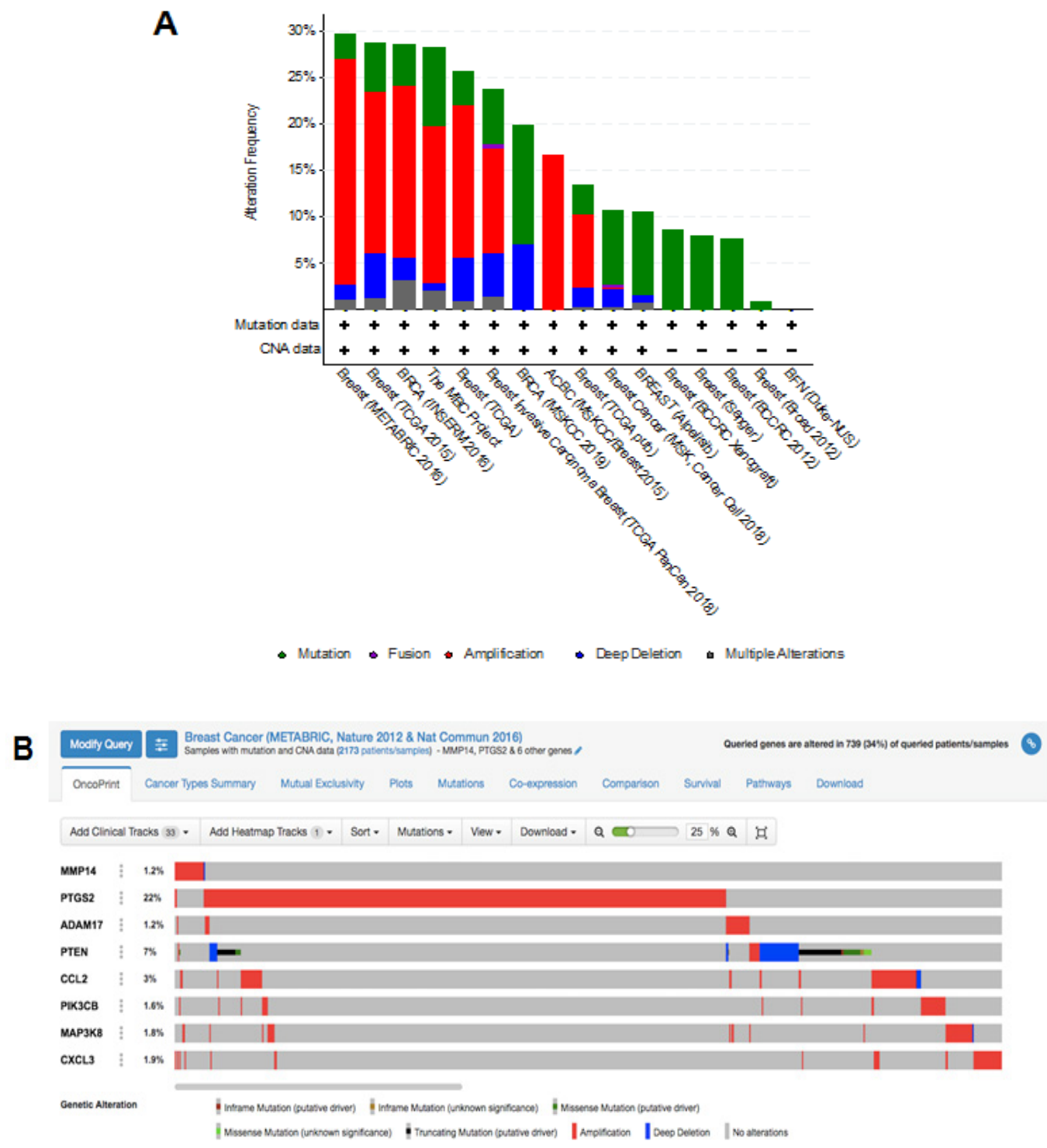

Figure 5. (A). Overview of genetic changes in MMP14, PTGS2, ADAM17, PTEN, CCL2, PIK3CB, MAP3K8, and $C X C L 3$ across 16 breast cancer studies, as analyzed by cBioportal. (B). Summary of alterations in MMP14, PTGS2, $A D A M 17, P T E N, C C L 2, P I K 3 C B, M A P 3 K 8$, and $C X C L 3$ across breast cancer patients using a study from Pereira et al., (2016).

synthase 2 or known as cyclooxygenase 2 (COX2), a key regulatory enzyme in the biosynthesis of prostaglandin E2 (Kozak et al., 2000). Overexpression of COX2 was found in about $40 \%$ of patients with invasive breast cancers (Singh et al., 2006). COX2 promotes bone metastasis of breast cancer cells through the upregulation of Interleukin-11 (IL-11) (Singh et al., 2006). Previous studies showed that COX2 promotes metastasis in breast cancer cells through several mechanisms, including infiltration by regulatory $\mathrm{T}$ cells (Tregs) (Karavitis and Zhang, 2013) and activation of the VEGF signaling pathway to trigger angiogenesis (Xu et al., 2014).

The crosstalk between COX 2 and TNF $\alpha$ was discussed in several previous studies. A previous study showed that TNF $\alpha$ induces the COX2 expression via activation of the NFkB signaling pathway in human colon cancer cells (Plummer et al., 1999), and activation of P38/MAPK pathway in colonic myofibroblast (Saini et al., 2016). $\mathrm{TNF} \alpha$ also promotes migration in colonic myofibroblast via activation of COX2 that is mediated by the P38/MAPK pathway (Saini et al., 2016). Moreover, TNF $\alpha$ also induces COX2 expression and subsequently increased PGE2 production and MMP9 expression in human fibroblast-like synovial cells (Alsousi et al., 2017).

ADAM17 encodes ADAM metallopeptidase domain 17 , a member of a disintegrin and metalloprotease domain family, and is also known as tumor necrosis factor (TNF)-converting enzyme (Li et al., 2019c). A previous study demonstrated that ADAM17 plays a role in the shedding of TNF $\alpha$ and membrane receptors protein, which involved in the biological process of proliferation, migration, and differentiation (Gutiérrez-López et al., 2011). On the other hand, ADAM17 is a pivotal enzyme for the generation of TNF- $\alpha$ in oral keratinocytes (Hirayama et al., 2017), and hemophilic arthropathy (Haxaire et al., 2018). Overexpression of ADAM17 promotes 5-fluorouracil resistance and metastasis in colorectal cancer cells via activation of the Notch signaling 
pathway (Li et al., 2018), and induces metastasis in gastric cancer through activation of Notch and Wnt signaling pathways (Li et al., 2019c). A review article discussed ADAM17 inhibitors for inflammation and cancer therapy (Kanda et al., 2017). However, the role of brazilin in ADAM17 activity and TNF $\alpha$ in promoting metastasis need to be clarified further.

PTEN encodes a phosphatidylinositol-3,4,5trisphosphate 3-phosphatase, a negative regulator of PI3K/Akt signaling pathway (Maehama et al., 2001). A previous study revealed the association between the loss of PTEN and metastasis in patients with endometrial cancer (Salvesen et al., 2002), prostate and breast cancer cells (Bandyopadhyay et al., 2004). Previous studies revealed the role and mechanisms of PTEN in breast cancer metastasis. PTEN inhibits proliferation, migration, and invasion in osteosarcoma cells by downregulating MMP9 and focal adhesion kinase (FAK) (Hu et al., 2014). Another study demonstrated that PTEN hampers invasion and metastasis of gastric cancer cells through the inhibition of the PI3K/NFkB pathway and preventing the DNA binding of NFkB on the FAK promoter (Zhang et al., 2014). Moreover, PTEN inhibits metastasis in breast cancer cells through the downregulation of WNT1 inducible signaling pathway protein 1 (WISP1) and lipocalin-2 (LCN2) (Chiang et al., 2016), and inactivation of NFkB signaling in non-small cell lung cancer cells (Akgun et al., 2019). A previous study showed that TNF $\alpha$ increased the expression of PTEN through activation of NFkB signaling in HL60 human leukemic cells (Lee et al., 2007). However, other studies demonstrated that PTEN inhibited the $\mathrm{PI} 3 \mathrm{~K} / \mathrm{AKT} / \mathrm{NF} \kappa \mathrm{B}$ signaling pathway that is induced by TNF $\alpha$ in human glioma cells (Koul et al., 2006), and prostate cancer cells (Lee et al., 2007). Further study of brazilin on the axis of PTEN-NFkB-TNF $\alpha$ in metastatic breast cancer cells is required.

The results of this present study showed genetic alterations in $7 \%$ of patients with metastatic breast cancer in the METABRIC study (Figure 4B). This result is supported by a recent study which demonstrated that mutation in PTEN leads to inactivation of its function as tumor suppressor genes in cancer (Luongo et al., 2019). A recent review article discussed the possibility of PTEN as a potential biomarker of lymph node metastasis of esophageal squamous cell carcinoma (Li et al., 2019b). Taken together, PTEN plays a pivotal role in breast cancer metastasis.

CCL2 encodes C-C motif chemokine ligand 2, also known as monocyte chemoattractant protein 1 (MCP1), a chemokine that regulates inflammatory processes (Daly and Rollins, 2003). Recent studies showed that CCL2 also regulates cancer metastasis. A previous study showed that CCL2 induces chemokine cascade signaling and subsequently promotes breast cancer metastasis by elevating retention of metastasis-associated macrophages (Kitamura et al., 2015). Another study demonstrated that CCL2 plays a role in macrophage recruitment that regulates lymphatic metastasis of bladder cancer (Chen et al., 2018). Increased CCL2 secretion was also found in the protumoral microenvironment induced by retinoblastoma inactivation (Li et al., 2019a).
The axis between TNF $\alpha$ signaling and CCL2 has also been studied, in which TNF $\alpha$ increases the expression of CCL2 in human proximal tubular epithelial cells via activation of MAPK signaling (Ho et al., 2008). A recent clinical trial of an inhibitor of CCL2, namely propagermanium, showed proper safety and efficacy in patients with metastatic breast cancer (Masuda et al., 2020). Taken together, CCL2 is a promising target for inhibiting breast cancer metastasis; however, the role of brazilin in metastasis-related to CCL2 and TNF $\alpha$ signaling needs to be explored further.

PIK3CB encodes phosphatidylinositol-4,5bisphosphate 3-kinase catalytic subunit beta $(\mathrm{PI} 3 \mathrm{~K} \beta)$, also known as $\mathrm{p} 110 \beta$, a protein involved in the PI3K/Akt signaling pathway (Pridham et al., 2018). Overexpression of PIK3CB was found in patients with colorectal carcinoma (Wen et al., 2014), non-small cell lung cancer (Xiong et al., 2017). Inhibition of PIK3CB with a specific inhibitor, reduced cell viability, and proliferation in glioblastoma (Pridham et al., 2018). In addition, PIK3CB, a significant regulator of the PI3K/Akt pathway, regulates metastasis in breast cancer cells (Hong et al., 2019). The $\mathrm{PI} 3 \mathrm{~K} / \mathrm{Akt}$ signaling is involved in the TNF $\alpha$ pathway in airway remodeling (Jude et al., 2012). Activation of PI3K/ Akt signaling increased the secretion of TNF $\alpha$ in activating macrophages (Huang et al., 2013). Nevertheless, the effects of brazilin on PIK3CB in metastatic breast cancer cells remain unclear.

$M A P 3 K 8$ encodes mitogen-activated protein kinase kinase kinase 8 , a member of serine/threonine kinase family (Paardekooper et al., 2018), which is involved in the $\mathrm{MAPK} / \mathrm{JNK} / \mathrm{p} 38$ and $\mathrm{NFkB}$ signaling pathway (Chorzalska et al., 2018). Overexpression of MAP3K8, also known as tumor progression locus 2 (TPL2), is correlated with poor prognosis and metastasis in patients with colorectal cancer (Pyo et al., 2018). Another study demonstrated that MAP3K 8 induces invasion and metastasis in renal cancer cells (Liu et al., 2016). MAP3K8 was also found to involve in the maintenance of thyroid cancer stem cells and thyroid cancer resistance to vemurafenib (Gianì et al., 2019). Genetic alterations in MAP3K8, including fusion and truncation, were found in $33 \%$ of patients with Spitz melanoma (Newman et al., 2019). Regarding the crosstalk with TNF $\alpha$ signaling, MAP3K8 was found to increase the expression of TNF $\alpha$ in monocyte-derived dendritic cells (Paardekooper et al., 2018). Accordingly, the role of MAP3K 8 in metastatic breast cancer and the effect of brazilin on MAP3K8 need to be explored in the future study.

CXCL3 encodes C-X-C motif chemokine ligand 3, a member of the CXC subfamily of chemokines, also known as a growth-related oncogene, is a potent neutrophil chemoattractant that regulates inflammation (Smith et al., 2005). The upregulation of CXCL3 was found in metastatic breast cancer and posses a potential therapeutic target (See et al., 2014). Other studies showed that CXCL3 is involved in migration and invasion of trophoblasts in the pathogenesis of preeclampsia (Wang et al., 2018) and is involved in the proliferation and migration of prostate cancer cells (Xin et al., 2018). A previous study demonstrated that CXCL3 is upregulated upon treatment 
of TNF $\alpha$ in adipocytes (Kusuyama et al., 2016). In addition, TNF $\alpha$ induced the upregulation of CXCL3 and its receptor in A498 renal cancer cells (Sun et al., 2016). Taken together, CXCL3 and its signaling is a potential target for preventing metastatic breast cancer. However, the effect of brazilin on CXCL3 signaling related to TNF $\alpha$ in metastatic breast cancer remains elusive.

Previous studies have demonstrated the effect of brazilin on TNF $\alpha$ signaling and PB. Brazilin suppressed the production of TNF $\alpha$ in lipopolysaccharide-induced RAW264.7 macrophages cells (Hu et al., 2009). Brazilin inhibited $\mathrm{TNF} \alpha$-induced $\mathrm{NFkB}$ signaling by targeting IKK in 293/IL-1R/TLR4 cells (Jeon et al., 2014). Brazilin was shown to decrease high glucose-induced vascular inflammatory through the inhibition of NFKB activation in HUVEC cells (Jayakumar et al., 2014). Brazilin was also found to decrease the expression of TNF $\alpha$ in mice with type-II collagen-induced arthritis (Jung et al., 2015) and to decrease the expression of COX2 and TNF $\alpha$, and inhibit ERK/NFkB signaling in RANKL-stimulated RAW264.7 cells (Kim et al., 2015). Recently, a study showed that brazilin possesses anti-inflammatory activity in $\mathrm{TNF} \alpha-$ induced psoriasis dermatitis by downregulating ERK/ JNK/p38 and NFkB signaling (Choi and Hwang, 2019). Further study of the effects brazilin on TNF $\alpha$ signaling and PB in metastatic breast cancer cells is required.

This present study revealed the new target genes and the mechanism of brazilin in inhibiting metastasis in breast cancer using a bioinformatics approach. This study identified eight new potential targets of brazilin for inhibiting metastatic breast cancer, including $M M P 14$, PTGS2, ADAM17, PTEN, CCL2, PIK3CB, MAP3K8, and $C X C L 3$. In addition, brazilin possibly inhibits metastatic breast cancer through inhibition of the TNF $\alpha$ signaling. The study results need to be validated with in vitro and in vivo studies to strengthen scientific evidence of the use of brazilin in inhibition of breast cancer metastasis.

\section{Acknowledgements}

The authors thank BPP UGM for their writing assistance.

\section{Author contribution}

AH-conception and design of the study, acquisition, analysis and interpretation of data, drafting and revising the article and final approval of the version to be published, HP-acquisition and analysis of data, drafting the article and final approval of the version to be published.

\section{Availability of material}

The datasets analysed during the present study are online available in the public database.

\section{Ethics approval and consent to participate \\ Not applicable.}

\section{Consent for publication \\ Not applicable.}

\section{Confict of interest}

The authors declare no conflict of interest.

\section{References}

Akgun S, Kucuksayan H, Ozes ON, et al (2019). NF-кBInduced Upregulation of miR-548as-3p Increases Invasion of NSCLC by Targeting PTEN. Anticancer Agents Med Chem, 19, 1058-68.

Alsousi A, Siddiqui S, Igwe O (2017). Cytokine-mediated differential regulation of cyclooxygenase-2, high mobility group box 1 protein and matrix metalloproteinase-9 expression in fibroblast-like synovial cells. J Clin Exp Pharmacol, 7.

Bandyopadhyay S, Pai SK, Hirota S, et al (2004). PTEN upregulates the tumor metastasis suppressor gene Drg-1 in prostate and breast cancer. Cancer Res, 64, 7655-60.

Cerami E, Gao J, Dogrusoz U, et al (2012). The cBio cancer genomics portal: an open platform for exploring multidimensional cancer genomics data. Cancer Discov, 2, 401-4.

Chen C, He W, Huang J, et al (2018). LNMAT1 promotes lymphatic metastasis of bladder cancer via CCL2 dependent macrophage recruitment. Nat Commun, 9, 3826.

Chiang KC, Hsu SY, Lin SJ, et al (2016). PTEN insufficiency increases breast cancer cell metastasis in vitro and in vivo in a Xenograft Zebrafish model. Anticancer Res, 36, 3997-4005.

Chin CH, Chen SH, Wu HH, et al (2014). cytoHubba: identifying hub objects and sub-networks from complex interactome. BMC Syst Biol, 8, S11.

Choi DH, Hwang HS (2019). Anti-inflammation activity of brazilin in TNF- $\alpha$ induced human psoriasis dermatitis skin model. Appl Biol Chem, 62, 46.

Chorzalska A, Ahsan N, Rao RSP, et al (2018). Overexpression of Tpl2 is linked to imatinib resistance and activation of MEK-ERK and NF- $\kappa B$ pathways in a model of chronic myeloid leukemia. Mol Oncol, 12, 630-47.

Cui G, Cai F, Ding Z, et al (2019). MMP14 predicts a poor prognosis in patients with colorectal cancer. Hum Pathol, 83, 36-42.

Daly C, Rollins BJ (2003). Monocyte chemoattractant protein-1 (CCL2) in inflammatory disease and adaptive immunity: therapeutic opportunities and controversies. Microcirculation, 10, 247-57.

Fleishman G, Yap HY, Murphy WK, et al (1983). Phase II trial of acivicin in advanced metastatic breast cancer. Cancer Treat Rep, 67, 843-4.

Fragomeni SM, Sciallis A, Jeruss JS (2018). Molecular subtypes and local-regional control of breast cancer. Surg Oncol Clin N Am, 27, 95-120.

Gao J, Aksoy BA, Dogrusoz U, et al (2013). Integrative analysis of complex cancer genomics and clinical profiles using the cBioPortal. Sci Signal, 6, pl1.

Gianì F, Russo G, Pennisi M, et al (2019). Computational modeling reveals MAP3K8 as mediator of resistance to vemurafenib in thyroid cancer stem cells. Bioinformatics, 35, 2267-75.

Griffin C, McNulty J, Pandey S (2011). Pancratistatin induces apoptosis and autophagy in metastatic prostate cancer cells. Int J Oncol, 38, 1549-56.

Gutiérrez-López MD, Gilsanz A, Yáñez-Mó M, et al (2011). The sheddase activity of ADAM17/TACE is regulated by the tetraspanin CD9. Cell Mol Life Sci, 68, 3275-92.

Ham B, Fernandez MC, D'Costa Z, et al (2016). The diverse roles of the TNF axis in cancer progression and metastasis. Trends in cancer research, 11, 1-27.

Hasinoff BB, Creighton AM, Kozlowska H, et al (1997). 
Mitindomide Is a Catalytic Inhibitor of DNA Topoisomerase II That Acts at the Bisdioxopiperazine Binding Site. $\mathrm{Mol}$ Pharmacol, 52, 839.

Haxaire C, Hakobyan N, Pannellini T, et al (2018). Bloodinduced bone loss in murine hemophilic arthropathy is prevented by blocking the iRhom2/ADAM17/TNF- $\alpha$ pathway. Blood, 132, 1064-74.

Hirayama A, Awano S, Seta Y, et al (2017). ADAM17 regulates TNF- $\alpha$ expression upon lipopolysaccharide stimulation in oral keratinocytes. Biomed Res, 38, 157-65.

Ho AW, Wong CK, Lam CW (2008). Tumor necrosis factor-alpha up-regulates the expression of CCL2 and adhesion molecules of human proximal tubular epithelial cells through MAPK signaling pathways. Immunobiology, 213, 533-44.

Hong BS, Ryu HS, Kim N, et al (2019). Tumor suppressor miRNA-204-5p regulates growth, metastasis, and immune microenvironment remodeling in breast cancer. Cancer Res, 79, 1520-34.

$\mathrm{Hu}$ CM, Liu YH, Cheah KP, et al (2009). Heme oxygenase-1 mediates the inhibitory actions of brazilin in RAW264.7 macrophages stimulated with lipopolysaccharide. J Ethnopharmacol, 121, 79-85.

$\mathrm{Hu} \mathrm{Y}, \mathrm{Xu} \mathrm{S}$, Jin W, et al (2014). Effect of the PTEN gene on adhesion, invasion and metastasis of osteosarcoma cells. Oncol Rep, 32, 1741-7.

Huang JB, Ding Y, Huang DS, et al (2013). Inhibition of the PI3K/AKT pathway reduces tumor necrosis factor-alpha production in the cellular response to wear particles in vitro. Artif Organs, 37, 298-307.

Jayakumar T, Chang CC, Lin SL, et al (2014). Brazilin ameliorates high glucose-induced vascular inflammation via inhibiting ROS and CAMs production in human umbilical vein endothelial cells. Biomed Res Int, 2014, 403703.

Jenie RI, Handayani S, Susidarti RA, et al (2018). The cytotoxic and antimigratory activity of Brazilin-doxorubicin on MCF7/HER2 cells. Adv Pharm Bull, 8, 507-16.

Jeon J, Lee JH, Park KA, et al (2014). Brazilin selectively disrupts proximal IL-1 receptor signaling complex formation by targeting an IKK-upstream signaling components. Biochem Pharmacol, 89, 515-25.

Jia Y, Tong X, Fan J (2019). Effect of brazilin on apoptosis and autophagy of tongue cancer Tca8113 cells and its molecular mechanism. Nan Fang Yi Ke Da Xue Xue Bao, 39, 351-6.

Jude JA, Tirumurugaan KG, Kang BN, et al (2012). Regulation of CD38 expression in human airway smooth muscle cells: role of class I phosphatidylinositol 3 kinases. Am J Respir Cell Mol Biol, 47, 427-35.

Jung EG, Han KI, Hwang SG, et al (2015). Brazilin isolated from Caesalpinia sappan L. inhibits rheumatoid arthritis activity in a type-II collagen induced arthritis mouse model. $B M C$ Complement Altern Med, 15, 124.

Kanda H, Kobayashi K, Yamanaka H, et al (2017). Microglial TNF $\alpha$ induces COX2 and PGI2 synthase expression in spinal endothelial cells during neuropathic pain. eNeuro, 4 , ENEURO.0064-17.2017.

Karavitis J, Zhang M (2013). COX2 regulation of breast cancer bone metastasis. Oncoimmunology, 2, e23129.

Kasurinen A, Tervahartiala T, Laitinen A, et al (2018). High serum MMP-14 predicts worse survival in gastric cancer. PLoS One, 13, e0208800.

Kawabata H, Azuma K, Ikeda K, et al (2017). TRIM44 is a poor prognostic factor for breast cancer patients as a modulator of NF-kB signaling. Int J Mol Sci, 18.

Kim B, Kim SH, Jeong SJ, et al (2012). Brazilin induces apoptosis and $\mathrm{G} 2 / \mathrm{M}$ arrest via inactivation of histone deacetylase in multiple myeloma U266 cells. J Agric Food Chem, 60, 9882-9.
Kim J, Lee HK, Chang TS, et al (2015). Inhibitory effect of brazilin on osteoclast differentiation and its mechanism of action. Int Immunopharmacol, 29, 628-34.

Kitamura T, Qian BZ, Soong D, et al (2015). CCL2-induced chemokine cascade promotes breast cancer metastasis by enhancing retention of metastasis-associated macrophages. $J$ Exp Med, 212, 1043-59.

Koul D, Takada Y, Shen R, et al (2006). PTEN enhances TNFinduced apoptosis through modulation of nuclear factorkappaB signaling pathway in human glioma cells. Biochem Biophys Res Commun, 350, 463-71.

Kozak KR, Rowlinson SW, Marnett LJ (2000). Oxygenation of the endocannabinoid, 2-arachidonylglycerol, to glyceryl prostaglandins by cyclooxygenase-2. J Biol Chem, 275, 33744-9.

Kusuyama J, Komorizono A, Bandow K, et al (2016). CXCL3 positively regulates adipogenic differentiation. J Lipid Res, 57, 1806-20.

Larsson A-M, Roxå A, Leandersson K, et al (2019). Impact of systemic therapy on circulating leukocyte populations in patients with metastatic breast cancer. Sci Rep, 9, 13451.

Lee AY, Park W, Kang T-W, et al (2018). Network pharmacologybased prediction of active compounds and molecular targets in Yijin-Tang acting on hyperlipidaemia and atherosclerosis. J Ethnopharmacol, 221, 151-9.

Lee DY, Lee MK, Kim GS, et al (2013). Brazilin inhibits growth and induces apoptosis in human glioblastoma cells. Molecules, 18, 2449-57.

Lee E, Ouzounova M, Piranlioglu R, et al (2019). The pleiotropic effects of TNF $\alpha$ in breast cancer subtypes is regulated by TNFAIP3/A20. Oncogene, 38, 469-82.

Lee YR, Yu HN, Noh EM, et al (2007). TNF-alpha upregulates PTEN via NF-kappaB signaling pathways in human leukemic cells. Exp Mol Med, 39, 121-7.

Lefebvre C, Bachelot T, Filleron T, et al (2016). Mutational profile of metastatic breast cancers: A Retrospective Analysis. PLoS Med, 13, e1002201.

Lehmann W, Edgar CM, Wang K, et al (2005). Tumor necrosis factor alpha (TNF-alpha) coordinately regulates the expression of specific matrix metalloproteinases (MMPS) and angiogenic factors during fracture healing. Bone, 36, 300-10.

Li DD, Zhao CH, Ding HW, et al (2018). A novel inhibitor of ADAM17 sensitizes colorectal cancer cells to 5-Fluorouracil by reversing Notch and epithelial-mesenchymal transition in vitro and in vivo. Cell Prolif, 51, e12480.

Li F, Kitajima S, Kohno S, et al (2019a). Retinoblastoma inactivation induces a protumoral microenvironment via enhanced CCL2 secretion. Cancer Res, 79, 3903-15.

Li J, Qi Z, Hu YP, et al (2019b). Possible biomarkers for predicting lymph node metastasis of esophageal squamous cell carcinoma: a review. $J$ Int Med Res, 47, 544-56.

Li W, Wang D, Sun X, et al (2019c). ADAM17 promotes lymph node metastasis in gastric cancer via activation of the Notch and Wnt signaling pathways. Int $J$ Mol Med, 43, 914-26.

Liu F, Chen N, Xiao R, et al (2016). miR-144-3p serves as a tumor suppressor for renal cell carcinoma and inhibits its invasion and metastasis by targeting MAP3K8. Biochem Biophys Res Commun, 480, 87-93.

Liu Y-L, Chou C-K, Kim M, et al (2019). Assessing metastatic potential of breast cancer cells based on EGFR dynamics. Sci Rep, 9, 3395.

Luongo F, Colonna F, Calapà F, et al (2019). PTEN tumorsuppressor: The dam of stemness in cancer. Cancers, 11, 1076.

Lyu Y, Xu X, Yun J, et al (2017). [TNF- $\alpha$ regulates the proliferation of human breast cancer cells via regulation of 
ceramide content]. Xi Bao Yu Fen Zi Mian Yi Xue Za Zhi, 33, 1303-9.

Maehama T, Taylor GS, Dixon JE (2001). PTEN and myotubularin: novel phosphoinositide phosphatases. Annu Rev Biochem, 70, 247-79.

Mahmoud N, Saeed MEM, Sugimoto Y, et al (2018). Cytotoxicity of nimbolide towards multidrug-resistant tumor cells and hypersensitivity via cellular metabolic modulation. Oncotarget, 9, 35762-79.

Masuda T, Noda M, Kogawa T, et al (2020). Phase I dose-escalation trial to repurpose propagermanium, an oral CCL2 inhibitor, in patients with breast cancer. Cancer Sci, 111, 924-31.

Monks A, Scudiero DA, Johnson GS, et al (1997). The NCI anti-cancer drug screen: a smart screen to identify effectors of novel targets. Anticancer Drug Des, 12, 533-41.

Naik Bukke A, Nazneen Hadi F, Babu KS, et al (2018). In vitro studies data on anticancer activity of Caesalpinia sappan L. heartwood and leaf extracts on MCF7 and A549 cell lines. Data Brief, 19, 868-77.

Newman S, Pappo A, Raimondi S, et al (2019). Pathologic characteristics of Spitz Melanoma with MAP3K8 fusion or truncation in a pediatric cohort. Am J Surg Pathol, 43, 1631-7.

Nirmal NP, Rajput MS, Prasad RGSV, et al (2015). Brazilin from Caesalpinia sappan heartwood and its pharmacological activities: A review. Asian Pac J Trop Med, 8, 421-30.

Paardekooper LM, Bendix MB, Ottria A, et al (2018). Hypoxia potentiates monocyte-derived dendritic cells for release of tumor necrosis factor $\alpha$ via MAP3K8. Biosci Rep, 38.

Plummer SM, Holloway KA, Manson MM, et al (1999). Inhibition of cyclo-oxygenase 2 expression in colon cells by the chemopreventive agent curcumin involves inhibition of NF-kappaB activation via the NIK/IKK signalling complex. Oncogene, 18, 6013-20.

Poster DS, Bruno S, Penta J, et al (1981). Acivicin. An antitumor antibiotic. Cancer Clin Trials, 4, 327-30.

Pridham KJ, Le L, Guo S, et al (2018). PIK3CB/p110 $\beta$ is a selective survival factor for glioblastoma. Neurooncology, 20, 494-505.

Pyo J-S, Park MJ, Kim C-N (2018). TPL2 expression is correlated with distant metastasis and poor prognosis in colorectal cancer. Hum Pathol, 79, 50-6.

Qiu S, Hu W, Ma Q, et al (2019). TIPE1 suppresses the invasion and migration of breast cancer cells and inhibits epithelialto-mesenchymal transition primarily via the ERK signaling pathway. Acta Biochim Biophys Sin (Shanghai), 51, 1008-15.

Rivera E, Gomez H (2010). Chemotherapy resistance in metastatic breast cancer: the evolving role of ixabepilone. Breast Cancer Res, 12, S2.

Roy S, Maity P (2007). Modulation of metastatic potential of B16F10 melanoma cells by acivicin: synergistic action of glutaminase and potentiation of cisplatin cytotoxicity. Asian Pac J Cancer Prev, 8, 301-6.

Saini S, Liu T, Yoo J (2016). TNF- $\alpha$ stimulates colonic myofibroblast migration via COX-2 and Hsp27. J Surg Res, 204, 145-52.

Salvesen HB, Stefansson I, Kalvenes MB, et al (2002). Loss of PTEN expression is associated with metastatic disease in patients with endometrial carcinoma. Cancer, 94, 2185-91.

Sampedro F, Partika J, Santalo P, et al (1994). Liposomes as carriers of different new lipophilic antitumour drugs: a preliminary report. J Microencapsul, 11, 309-18.

Scheff NN, Ye Y, Bhattacharya A, et al (2017). Tumor necrosis factor alpha secreted from oral squamous cell carcinoma contributes to cancer pain and associated inflammation. Pain, 158, 2396-409.
See AL, Chong PK, Lu SY, et al (2014). CXCL3 is a potential target for breast cancer metastasis. Curr Cancer Drug Targets, 14, 294-309.

Shafei A, El-Bakly W, Sobhy A, et al (2017). A review on the efficacy and toxicity of different doxorubicin nanoparticles for targeted therapy in metastatic breast cancer. Biomed Pharmacother, 95, 1209-18.

Shuman Moss LA, Jensen-Taubman S, Stetler-Stevenson WG (2012). Matrix metalloproteinases: changing roles in tumor progression and metastasis. Am J Pathol, 181, 1895-9.

Singh B, Berry JA, Shoher A, et al (2006). COX-2 induces IL-11 production in human breast cancer cells. J Surg Res, 131, 267-75.

Singh K, Roy M, Prajapati P, et al (2019). NLRX1 regulates TNF- $\alpha$-induced mitochondria-lysosomal crosstalk to maintain the invasive and metastatic potential of breast cancer cells. Biochim Biophys Acta Mol Basis Dis, 1865, 1460-76.

Smith DF, Galkina E, Ley K, et al (2005). GRO family chemokines are specialized for monocyte arrest from flow. Am J Physiol Heart Circ Physiol, 289, H1976-84.

Sun K-H, Sun G-H, Wu Y-C, et al (2016). TNF- $\alpha$ augments CXCR2 and CXCR3 to promote progression of renal cell carcinoma. J Cell Mol Med, 20, 2020-8.

Szklarczyk D, Franceschini A, Wyder S, et al (2015). STRING v10: protein-protein interaction networks, integrated over the tree of life. Nucleic Acids Res, 43, D447-52.

Telli ML, Carlson RW (2009). First-line chemotherapy for metastatic breast cancer. Clin Breast Cancer, 9, S66-72.

Wang H, Wang T, Dai L, et al (2018). Effects of CXCL3 on migration, invasion, proliferation and tube formation of trophoblast cells. Placenta, 66, 47-56.

Wang J, Vasaikar S, Shi Z, et al (2017). WebGestalt 2017: a more comprehensive, powerful, flexible and interactive gene set enrichment analysis toolkit. Nucleic Acids Res, 45, W130-w7.

Welch DR, Hurst DR (2019). Defining the hallmarks of metastasis. Cancer Res, 79, 3011-27.

Wen F, He S, Sun C, et al (2014). PIK3CA and PIK3CB expression and relationship with multidrug resistance in colorectal carcinoma. Int J Clin Exp Pathol, 7, 8295-303.

Wolczyk D, Zaremba-Czogalla M, Hryniewicz-Jankowska A, et al (2016). TNF- $\alpha$ promotes breast cancer cell migration and enhances the concentration of membrane-associated proteases in lipid rafts. Cell Oncol (Dordr), 39, 353-63.

Wu W, Jingbo S, Xu W, et al (2018). S-trityl-L-cysteine, a novel Eg5 inhibitor, is a potent chemotherapeutic strategy in neuroblastoma. Oncol Lett, 16, 1023-30.

Wu Y-d, Zhou B (2010). TNF- $\alpha /$ NF- $\kappa$ B/Snail pathway in cancer cell migration and invasion. Br J Cancer, 102, 639-44.

Xin H, Cao Y, Shao ML, et al (2018). Chemokine CXCL3 mediates prostate cancer cells proliferation, migration and gene expression changes in an autocrine/paracrine fashion. Int Urol Nephrol, 50, 861-8.

Xiong Y, Qu L, Li D, et al (2017). [Clinical Significance and Mechanism of PI3K p110 $\beta$ Overexpression in Non-small Cell Lung Cancer]. Zhongguo Fei Ai Za Zhi, 20, 808-16.

$\mathrm{Xu}$ L, Stevens J, Hilton MB, et al (2014). COX-2 inhibition potentiates antiangiogenic cancer therapy and prevents metastasis in preclinical models. Sci Transl Med, 6, 242 ra84.

Yousefi M, Nosrati R, Salmaninejad A, et al (2018). Organspecific metastasis of breast cancer: molecular and cellular mechanisms underlying lung metastasis. Cell Oncol (Dordr), 41, 123-40.

Yu PF, Huang Y, Han YY, et al (2017). TNF $\alpha$-activated mesenchymal stromal cells promote breast cancer metastasis by recruiting CXCR2(+) neutrophils. Oncogene, 36, 482-90. 
Zhang LL, Liu J, Lei S, et al (2014). PTEN inhibits the invasion and metastasis of gastric cancer via downregulation of FAK expression. Cell Signal, 26, 1011-20.

Zhang T, Fan X, Song L, et al (2015). c-Fos is involved in inhibition of human bladder carcinoma $\mathrm{T} 24$ cells by brazilin. IUBMB Life, 67, 175-81.

Zhang Z, Lin G, Yan Y, et al (2018). Transmembrane TNF-alpha promotes chemoresistance in breast cancer cells. Oncogene, 37, 3456-70.

Zhao P, Zhang Z (2018). TNF- $\alpha$ promotes colon cancer cell migration and invasion by upregulating TROP-2. Oncol Lett, 15, 3820-7.

\section{(c) (1) ()}

This work is licensed under a Creative Commons AttributionNon Commercial 4.0 International License. 